\title{
A Novel Cooperative Spectrum Sensing scheme with primary user protection
}

\author{
J.Geetha Ramani \\ Assistant Professor, Department of ECE, \\ SNS College of Technology, Coimbatore. \\ Dr.K.Geetha \\ Professor and Head, Department of EEE, \\ Karpagam Institute of Technology, Coimbatore.
}

\begin{abstract}
Cognitive Radio is a technology which is capable of optimizing the radio spectrum utilization. Primary User Emulation Attack (PUEA) is one of security problems that physical layer needs facing, which has great threat to spectrum sensing. Also optimal bandwidth should be allocated for cognitive users for required throughput efficiency. A novel cooperative sensing scheme is proposed with energy detection. The sensing information of various cognitive users are combined at fusion center using 3 bit hard combination scheme. Simulation results show the effectiveness of the proposed system in cooperative spectrum sensing with PUEA.
\end{abstract}

Keywords- COGNITIVE RADIO-PUEA- COOPERATIVE SPECTRUM SENSING-HARD COMBINATION SCHEME.

\section{INTRODUCTION}

Cognitive radio is a technology for wireless communications in which a user flexibly changes their transmitting or receiving parameters to achieve more efficient communication performance without interfering with primary or secondary users [1]. Under the assumption of cognitive radio, wireless system should be sufficiently smart to recognize the variation of the environment and dynamically adjust its parameters to adapt the alternations.

The security issues are the major problem in wireless networks. The potential security vulnerabilities and mitigation countermeasures are surveyed in [2] [3] [4]. In [4], Qiwei Zhang et. al exemplify intentional and unintentional attacks in cognitive radio networks and claim that the direct jamming can be significantly reduced by carefully designing the cognitive architecture.

Apart from the above work, one typical vulnerability of cognitive radio, called primary user emulation attack (PUEA) is identified in [5], which has been mentioned in many other literatures by the different forms of expression.

A selfish or malicious secondary user may preempt an idle frequency band by imitating the primary user and thus prevents other secondary users from accessing that band. Such a malicious behavior or attack is termed as primary user emulation attack (PUEA). The defense measurements involve as either locating the real position of attacker and primary user or increasing the trust level of the legitimated users in the network through public key encryption algorithm [7].

However, it is difficult to differentiate the locations of both attacker and actual primary user if they are much closed geographically and for the encryption scheme, secondary users should be capable of strictly synchronizing and demodulating primary signals, which is a rigorous requirement for the CR users. Although a variety of research efforts have been directed on the detection of PUEA, the cooperative spectrum sensing with the existence of PUEA is less investigated.

In proposed scheme, detection probability of primary user is maximized. As the PUEA is launched in a CR network using cooperative sensing technique, each secondary user receives the signals from the attacker and the primary user and sends its sensing information to a fusion center. The received signal (or the sensing information) is then optimally combined with hard bit combination scheme to maximize the detection probability with a constraint of false alarm probability.

\section{II.PRIMARY USER EMULATION ATTACK}

PUEA is one of security problems that physical layer needs facing, which has great threat to Spectrum Sensing. The primary user can utilize the authorized frequency band free of all times. Figure 1. Shows the PUEA attack in the cooperative spectrum sensing. The authorized frequency band turns into idle state when the primary user releases the resources, so the secondary users can attempt to access. When the presence of primary user detected 
by secondary user, it must switch over to other vacant channels in order to minimize the interference to primary user.

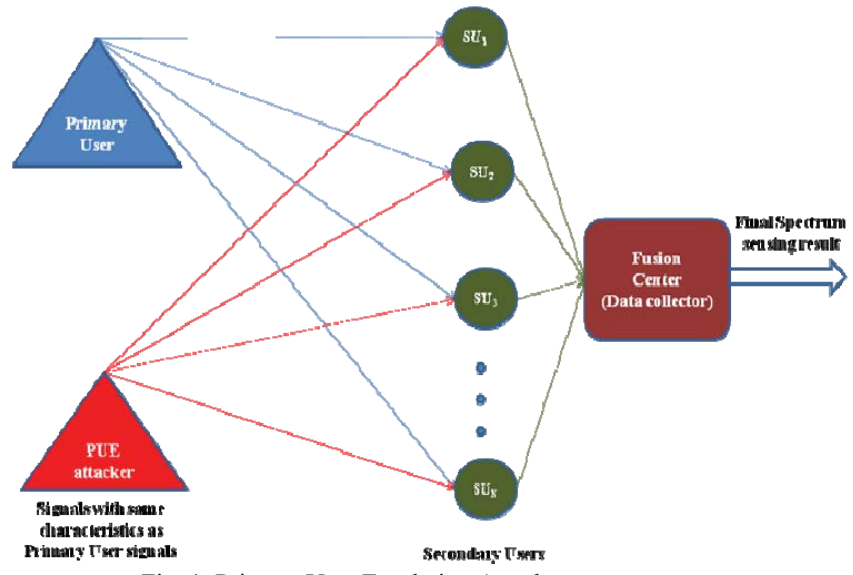

Fig. 1. Primary User Emulation Attack

III. ENERGY DETECTION

Energy detection is the signal detection mechanism using an energy detector (also known as radiometer) to specify the presence or absence of signal in the band [1].

In an energy detector, the received signal i.e., input signal is passed through a Band Pass Filter (BPF). The band pass filter reduces the noise bandwidth. Then it is passed through the squaring device to increase the signal strength. The output signal from the integrator is compared with a threshold value. If the signal value is greater than the predefined threshold, then the presence of primary user is detected [5]. Figure 2 shows a block diagram of an energy detector.

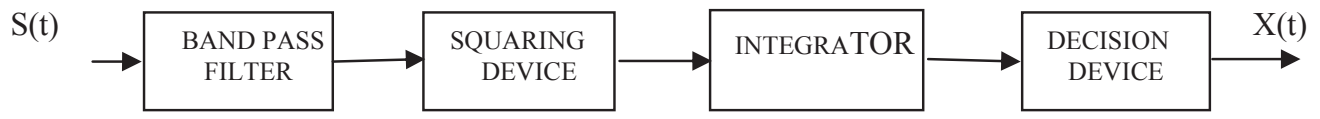

Fig. 2. Block Diagram of Energy Detector

IV. System Model

Let consider a cognitive network with $\mathrm{N}$ cognitive users ( detect PU in a spectrum by means of sensing process. The hypothesis problem for spectrum sensing with two hypotheses $\mathrm{H} 0$ and $\mathrm{H} 1$ can be formulated as

$$
\begin{array}{ll}
\mathrm{X}_{\mathrm{n}}(\mathrm{t})=\mathrm{w}_{\mathrm{n}}(\mathrm{t}) & : \mathrm{H}_{0} \\
\mathrm{X}_{\mathrm{n}}(\mathrm{t})=\mathrm{h}_{\mathrm{n}} * \mathrm{~s}(\mathrm{t})+\mathrm{w}_{\mathrm{n}}(\mathrm{t}) & : \mathrm{H}_{1}
\end{array}
$$

Where,

$$
\begin{aligned}
& s(t) \text { - Primary signal } \\
& w_{n}(t) \text { - Receiver noise of the CR user with zero mean and variance } \\
& \quad h_{n} \text { - Channel gain between primary user and } n^{\text {th }} C R \text { user }
\end{aligned}
$$




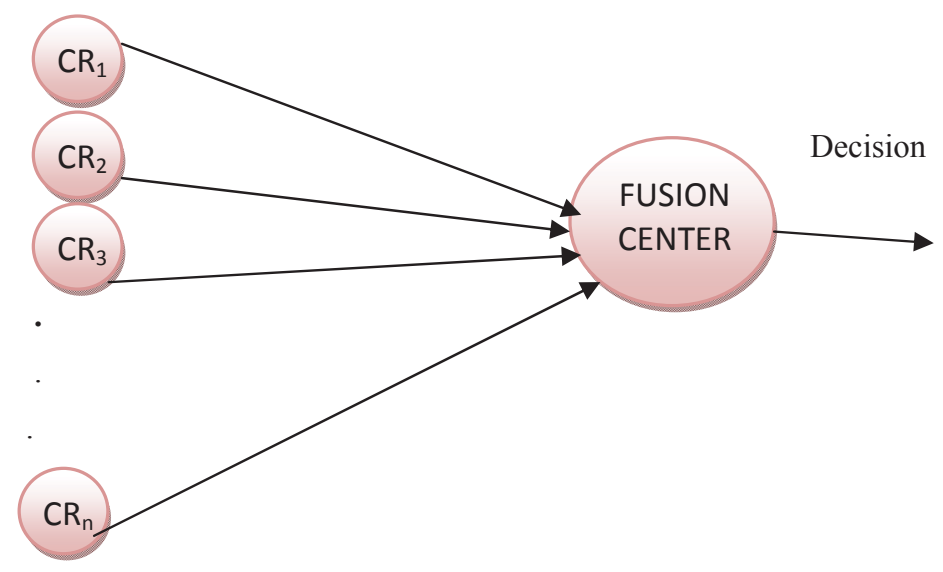

Fig. 3. Cooperative spectrum sensing

The information sensed by individual nodes about the presence of PU to the decision node. Using this cooperation, the effect of multipath propagation such as fading, shadowing can be minimized.

\section{PROPOSED COOPERATIVE SPECTRUM SENSING SCHEME}

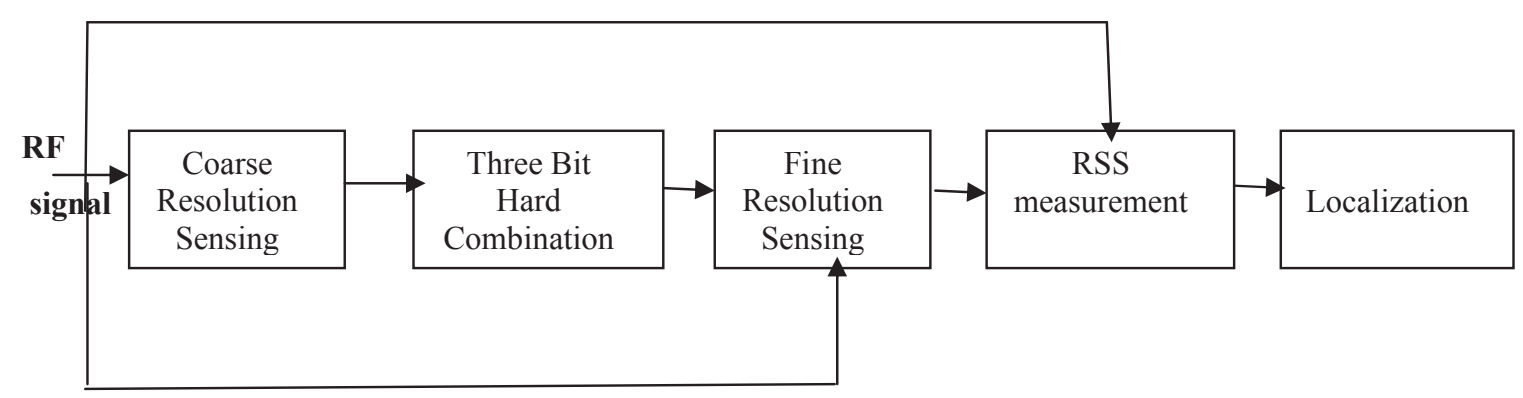

Fig. 4.Block diagram of cooperative spectrum sensing

In the proposed technique, the distance between a cognitive user and other users is calculated based on location coordinates as well as received power level. If the distance calculated with both these techniques matches, then the user is a trustworthy user. In other case, it would be considered as a malicious user.

Received Signal Strength (RSS) based localization methods are used to calculate the location. The waveletbased Multi Resolution Spectrum Sensing (MRSS) scheme is combined with RSS for spectrum sensing. The performance can be evaluated by calculating the Mean Square Error (MSE) to find the position and power can be estimated by average absolute Power Estimation Error (PEE).

MRSS is a kind of energy detector. The basis for MRSS is the sensing of a spectrum at two different resolutions.

1. Coarse resolution

2. Fine resolution

The decision node senses the entire spectrum using coarse resolution and determines seven thresholds. These values are used to divide the observation region in to eight different ranges. The threshold values are sending to all the nodes. The nodes except decision maker node perform the coarse resolution sensing and send the observed energy value as three bit information to the decision maker. Based on these values the decision maker determines, the region where fine resolution is to be applied in the spectrum. The decision maker also determines the nodes, which senses the highest energies on the spectrum bands apply fine resolution sensing. For finding locations, averaged RSS value from the nodes and RSS based localization scheme is used

\section{Combination Schemes}

Hard combination is the type of cooperative spectrum sensing scheme. In this scheme, the decision maker will take the decision based on the information collected from the other.

Algorithm 
1. Spectrum sensing process is carried by every user.

2. By comparing the sensing result with threshold, a binary decision can be taken about the presence of signal.

3. All the decisions are forwarded to decision node.

4. A final decision on whether the signal of interest is present or not will be made by the decision maker.

Fusion Rule

Each CR user makes its own decision concerning the presence of the PU and forwards the binary decision ( 1 or 0 ) to fusion center (FC) for data fusion.

Assuming uncorrelated decisions, the probability of detection at the fusion center is given by:

$$
E_{\mathrm{d}}=\sum_{\mathrm{n}}^{W}\left(\begin{array}{l}
M \\
k
\end{array}\right) E_{\mathrm{w} \cdot \mathrm{k}} \mathrm{k}\left(1-E_{\mathrm{d} k}\right)^{M \cdot k}
$$

where

Pd,i is the probability of detection for each individual node.

$\mathrm{M}$ denotes the number of users sensing the spectrum.

OR fusion Rule

Cooperative detection performance with this fusion rule can be evaluated by setting $\mathrm{n}=1$

$$
P_{d, O R=1-\left(1-P_{d i}\right)} M
$$

Majority fusion Rule

Cooperative detection performance with this fusion rule can be evaluated by setting $\mathrm{n}=\lfloor\mathrm{M} / 2\rfloor$

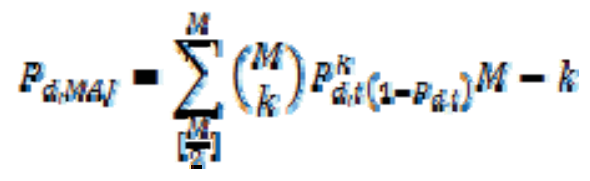

\section{AND fusion Rule}

The fusion center's decision is calculated by a logic AND of the received hard decision statistics. Cooperative detection performance with this fusion rule can be evaluated by setting $n=M$ in equation

$$
P_{d_{i} A M D} \cdot\left(P_{Q 1}\right)^{M}
$$

Three bit hard combination scheme

In the three-bit scheme, whole range of observed energy is divided in to eight regions. Seven thresholds are used to divide the eight regions. Each CR user sends three bit information to the fusion center. The three bit information gives the region where the observed energy fell.

The final decision is made by comparing this sum with a threshold. Using energy detector, the nth CR user will calculate the received energy as

$$
E_{n}=\sum_{N=1}^{n} X_{n}^{2}(t)
$$




\begin{tabular}{|cc} 
Energy & $(111)$ \\
\hline Region 7 & $(110)$ \\
\hline Region 6 & $(101)$ \\
\hline Region 5 & $\lambda_{6}$ \\
\hline Region 4 & $(100)$ \\
\hline Region 3 & $(011)$ \\
\hline Region 2 & $\lambda_{4}$ \\
\hline Region 1 & $(010)$ \\
\hline Region 0 & $(001)$ \\
\hline
\end{tabular}

Fig. 5. Energy Regions

For each region the weights are defined as

$$
\mathrm{W}_{0}=0, \mathrm{~W}_{1}=1, \mathrm{~W}_{2}=\mathrm{L}, \mathrm{W}_{3}=\mathrm{L}^{2}, \mathrm{~W}_{4}=\mathrm{L}^{3}, \mathrm{~W}_{5}=\mathrm{L}^{4}, \mathrm{~W}_{6}=\mathrm{L}^{7}, \mathrm{~W}_{7}=\mathrm{L}^{8} .
$$

Where $\mathrm{L}$ is the design parameter

The criterion used to identify the presence of user is

$$
\sum_{n=0}^{7} W_{n} N_{n} \geq L^{8}
$$

Where $N_{n}$ is the number of observed energies fall in to region $n$.

\section{RSS based Localization Scheme}

The ideal RSS value at the $\mathrm{j}^{\text {th }}$ node is equal to the ideal received power $P_{r, j}^{\text {ideal }}$ , Which is given by

$$
P_{r, j}^{\text {ideal }}=C_{j} \frac{P_{t}}{d_{j}^{\alpha}}, \quad j=1,2, \ldots M
$$

Where,

$P t$ - EIRP of the transmitter,

$C j$ - Constant representing the factors effecting RSS, such as antenna gain and Height

$\alpha$ - Path loss exponent

$d j$ - Distance between the transmitter and the $\mathrm{j}^{\text {th }}$ node, represented by

$$
d_{j}=\sqrt{\left(x-x_{j}\right)^{2}+\left(y-y_{j}\right)^{2}}
$$

$(x, y)$ - Real position of the transmitter , $\quad(x \mathrm{j}, \mathrm{y} \mathrm{j})$ - Position of the $j^{\text {th }}$ node.

RSS values are averaged using 


$$
P_{r, j}^{\text {ideal }} \approx \frac{1}{R} \sum_{i=1}^{R} P_{r, j i}
$$

Where,

$$
\text { R- Total No.of samples }
$$

$P_{r, j i}-\mathrm{i}^{\text {th }}$ sample RSS value at the $\mathrm{j}^{\text {th }}$ node in $\mathrm{dBm}$

Simulation is carried out in MATLAM v2011. Table I shows the important parameters for the simulation.

Table I Parameters for simulation

\begin{tabular}{|l|l|}
\hline PARAMETER & VALUE/RANGE \\
\hline Topology size & $2000 \mathrm{~m} \times 2000 \mathrm{~m}$ \\
\hline No.of PU transmitter & 1 \\
\hline No.of Cognitive users & 20 \\
\hline No.of samples & $200 \mathrm{to} 400$ \\
\hline PU transmit power & $30 \mathrm{dBm}$ \\
\hline SU transmit power & $10 \mathrm{dBm}, 20 \mathrm{dBm}$ \\
\hline Frequency & $31-130 \mathrm{MHz}$ \\
\hline
\end{tabular}

Figure 6 shows the trustworthiness of a user. If a SNR value increases then the trustworthiness increases. If the trustworthiness reaches to 1 , then conclude that the communication is with primary user and not with malicious user. As SNR value increases, the trustworthiness also increases and reaches to 1 when SNR is 5. Whereas, malicious user's trustworthiness remains constant at 0.6 , even though the SNR value increases.

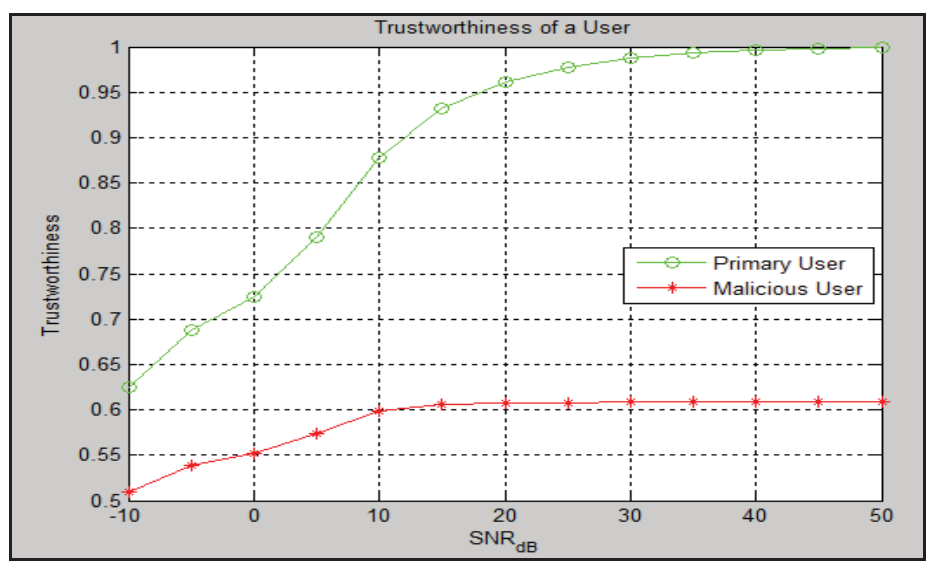

Fig .6. Trustworthiness of the user

Figure 7 shows the secondary users capacity per active Secondary Users(SU) for varying number of malicious users. When No. of users increases the secondary user capacity reduces. 


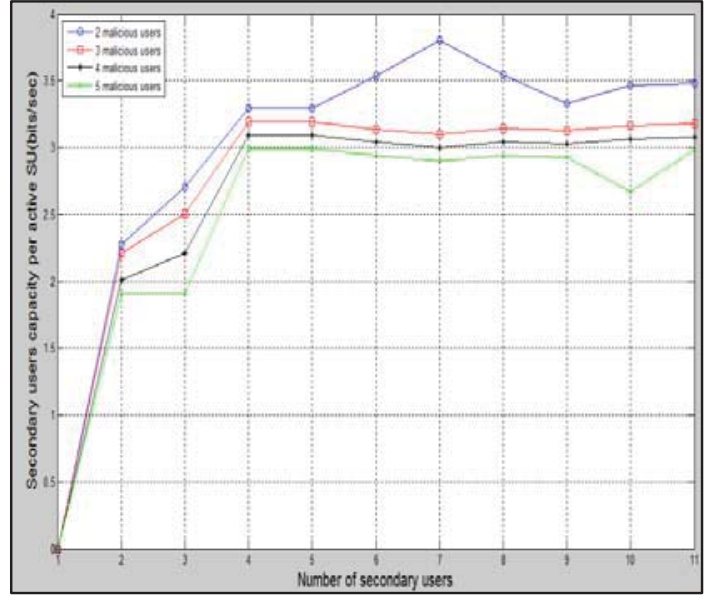

Fig.7.Secondary users capacity with different No. of malicious users

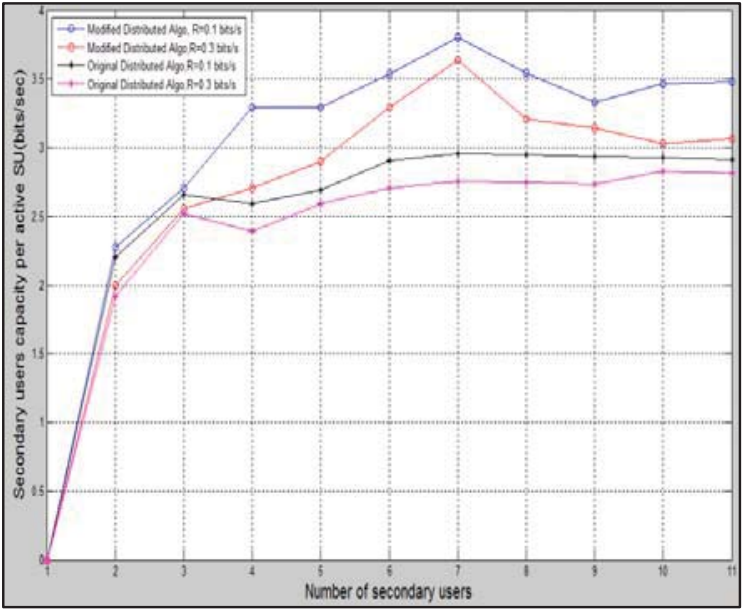

Fig.8.Comparison of secondary users capacity for original and modified algorithm with 2 malicious users

Figure 8 shows the secondary user's capacity per active SU for original and modified algorithm for different rates with 2 malicious users. Modified algorithm with $\mathrm{R}=0.1 \mathrm{bits} / \mathrm{sec}$ shows the good performance among all. The above results show that if the No. of malicious users is less, then the proposed scheme performs well on increasing secondary user's capacity.

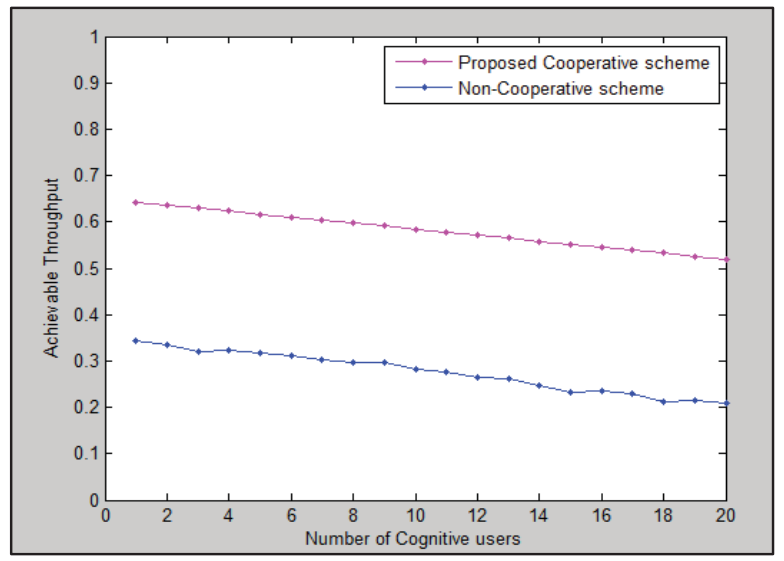

Fig. 9.Throughput comparison between cooperative and Cooperative

Non cooperative scheme

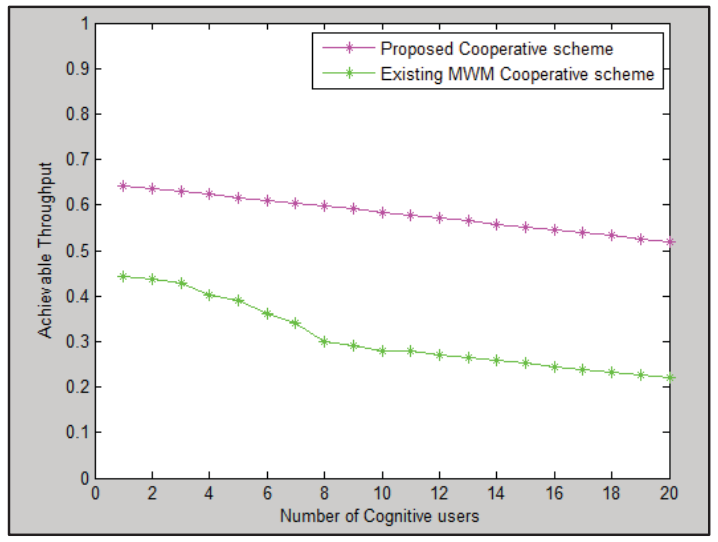

Fig.10.Throughput comparison for proposed

and existing MWM Cooperative scheme

Figure 9 and 10 shows the throughput for the proposed scheme with compared to non cooperative and existing schemes. The results show that there is 30\% improvement in throughput for the proposed scheme. When number of cognitive user increases

Figure 11 depicts the delay performance of the proposed system is reduced when compared to the existing system. The result show that there is $10 \%$ reduction in the delay when compared to the existing system. 


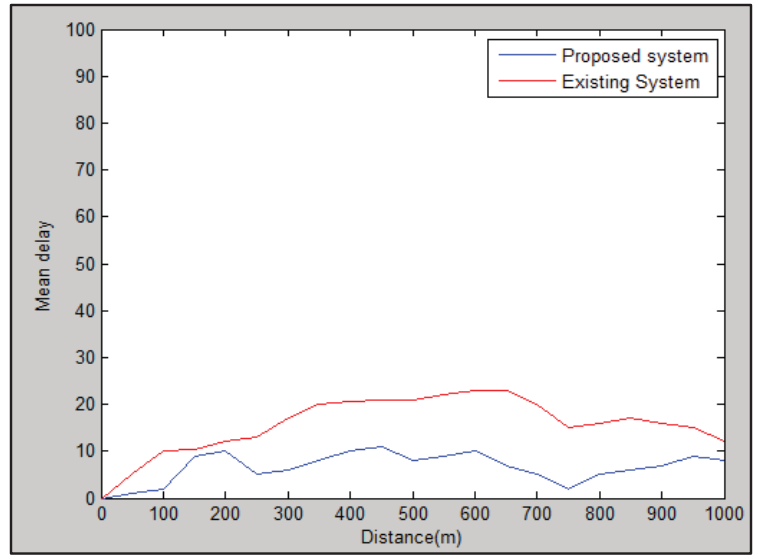

Fig.10. Detection delay performance comparison

\section{CONCLUSION}

The performance of cooperative based spectrum sensing schemes using energy detection with primary user protection is investigated using a simulation. The performance has been investigated via throughput and delay performance and is also investigated for different No. of secondary user's. From the observed results it is concluded that proposed cooperative spectrum sensing shows better performance.

\section{REFERENCES}

[1] Jun Ma, Geoffrey Ye Li, and Biing Hwang (Fred) Juang, "Signal processing in cognitive radio," Proceedings of the IEEE, Vol. 97, No. 5, pp. 805-823, 2009 .

[2] Nathan M. Neihart, Sumit Roy, and David J. Allstot, "A parallel, multi-resolution sensing technique for multiple antenna cognitive radios," Proc. of IEEE International Symposium on Circuits and Systems, pp. 2530-2533, 2007.

[3] J. Park, Y. Hur, T. J. Song, K. Kim, J. Lee, K. Lim, C.-H. Lee, H. S. Kim, and J. Laskar, "Implementation issues of a wideband multiresolution spectrum sensing (MRSS) technique for cognitive radio (CR) systems," Proc. of 1st International Conference on Cognitive Radio Oriented Wireless Networks and Communications, pp. 1-5, 2006.

[4] Qiwei Zhang, Andre B.J. Kokkeler and Gerard J.M. Smit, "An efficient multiresolution spectrum sensing method for cognitive radio," Proc. of Third International Conference on Communications and Networking in China, pp.,1226-1229, 2008.

[5] Sunghun Kim, Hyoungsuk Jeon, and Joongsoo Ma, "Robust localization with unknown transmission power for cognitive radio," Proc. of IEEE Military Communications Conference, pp. 1-6, 2007.

[6] Ying-Chang Liang, Yonghong Zeng, Anh Tuan Hoang, Chee Wei Ang, and Edward Peh, "Cognitive radio for wireless regional area networks," in Cognitive Radio Networks, Yang Xiao and Fei Hu, eds., pp. 3-35, Auerbach Pub., Boca Raton, Florida, 2008.

[7] Yun Wang, Xiaodong Wang, Demin Wang, and Dharma P. Agrawal, "Range-free localization using expected hop progress in wireless sensor networks," IEEE Transactions on Parallel and Distributed Systems, Vol. 20, No. 10, pp. 1540- 1552, 2009. 